\title{
The calm after the cytokine storm: lessons from the TGN1412 trial
}

\author{
E. William St. Clair
}

Departments of Medicine and Immunology, Duke University Medical Center, Durham, North Carolina, USA.

\begin{abstract}
In March 2006, a phase I study of the superagonistic anti-CD28 antibody TGN1412 caused a massive cytokine storm and multiorgan failure in six healthy human volunteers. Such a profound impact on the immune system was not predicted by preclinical animal studies. In a study from this issue of the JCI, Müller et al. treated rats with the superagonistic anti-CD28 antibody JJ316 and found that it rapidly induced a marked $\mathrm{T}$ cell lymphopenia by trapping $T$ cells in the spleen and lymph nodes (see the related article beginning on page 1405). This dramatic redistribution of $T$ cells simulated the profound $\mathrm{T}$ cell lymphopenia observed in human recipients of TGN1412. In contrast, JJ316 treatment in the rats did not reproduce the massive cytokine storm observed following TGN1412 administration to the human volunteers. These results point to similarities as well as differences between rodents and humans in the immunological effects of superagonistic anti-CD28 antibody treatment and raise further questions about how best to design preclinical studies that can better predict the risks of novel immunotherapeutics in humans.
\end{abstract}

A calm of careful retrospection has taken over the initial shock of learning about the devastating effects of a massive cytokine storm in six human volunteers who received a single infusion of the humanized monoclonal antibody TGN1412 (1). The key players in drug development are still taking stock of these events while contemplating strategies to prevent their recurrence. TGN1412, a superagonistic anti-human CD28 antibody (IgG4א), had passed the usual degree of preclinical testing, and similar reagents had shown efficacy in rodent models for treating autoimmune disease (2-4). However, TGN1412 had moved through early development on its way to a phase I trial without a deep understanding of the potential safety of this approach in humans. The superagonistic anti-CD28 antibody was able to uniquely activate $T$ cells in the absence of a concomitant TCR-mediated signal, which unlocked new avenues for immunotherapy but also the possibility of unknown dangers. We may conclude, in hindsight, that these unforeseen, serious adverse events

Nonstandard abbreviations used: ESG, Expert Scientific Group.

Conflict of interest: The author has received research support from Genentech.

Citation for this article: J. Clin. Invest. 118:1344-1347 (2008). doi:10.1172/JCI35382. might have been anticipated in light of the high-risk nature of TGN1412's molecular target and largely avoidable had the clinical protocol been designed with the appropriate risk-minimization strategies. In this issue of the JCI, Müller and colleagues (5) describe the effects of treatment with a superagonistic anti-CD28 antibody (JJ316) on the mechanisms of $\mathrm{T}$ cell redistribution and activation in the rat, providing additional insights into the immunological basis for what was observed in human subjects during the first few days after TGN1412 administration.

\section{High stakes and lurking dangers}

The story of TGN1412 ranks as a prime example of a drug development program led astray by the failure of sponsors, investigators, a clinical research organization, and regulators to ask crucial questions about the risks of an untested therapy with a novel mechanism of action. TGN1412 was in development for the treatment of B cell chronic lymphocytic leukemia, with the expectation that it would reverse the $\mathrm{T}$ cell deficiency in this disease, by inducing polyclonal expansion and activation of the $\mathrm{T}$ cell compartment. It was also hypothesized to be an expander of Tregs that might protect against the onslaught of autoimmune disease (reviewed in ref. 6). Much of the early preclinical work had been done in rats using JJ316, an antibody against rat $\mathrm{CD} 28$ that was functionally equivalent to TGN1412, and the same antibody used by Müller et al. in their current study (5). In rat models, treatment with JJ316 had been shown to ameliorate EAE and adjuvant arthritis through the induction of $\mathrm{CD} 4^{+} \mathrm{CD} 25^{+} \mathrm{FoxP}^{+}$Tregs with potent suppressive activity (4). These results provided a strong reason to believe that TGN1412 could become a successful first-in-class therapy with a novel mechanism of action.

However, unknown at the time was the precise mechanism by which TGN1412 activated $\mathrm{T}$ cells. The investigator brochure for TGN1412 surmised that the risk of developing a massive, TGN1412-induced cytokine storm was relatively low based on the results of TGN1412 testing in nonhuman primates (7). TGN1412 was known to react with CD28 from rhesus and cynomolgus monkeys, wherein it elicited a predictable, transient, and reversible expansion of $\mathrm{CD}^{+}$and $\mathrm{CD} 8^{+} \mathrm{T}$ cells and proliferation of $\mathrm{CD} 4^{+} \mathrm{CD} 25^{+} \mathrm{T}$ cells (7). According to the investigator brochure, relatively modest levels of proinflammatory cytokines were produced in cynomolgus monkeys when they were treated intravenously with four weekly doses of $5 \mathrm{mg} / \mathrm{kg}$ (low dose) or 50 $\mathrm{mg} / \mathrm{kg}$ (high dose) of TGN1412 (7). In these experiments, high-dose TGN1412 provoked moderate increases in the serum levels of IL-2, IL-5, and IL-6, with no significant changes in the serum levels of IL-4, IFN- $\gamma$, or TNF- $\alpha$. Treatment at the lower dose resulted in only weak IL-5 and IL-6 responses. Thus, $0.1 \mathrm{mg} / \mathrm{kg}$, or a 500 th of the no-observed-adverse-effect level in nonhuman primates $(50 \mathrm{mg} / \mathrm{kg})$, was deemed to be an appropriate starting dose for the phase I trial.

\section{The Northwick Park tragedy: an unforeseen storm}

On March 13, 2006, TeGenero AG initiated its first-in-human clinical trial of TGN1412 at the Northwick Park and St. Mark's Hospital, London, United Kingdom, under the 
Issues raised in the ESG report related to first-in-human studies

\section{Preclinical and early clinical development}

Adequacy of science behind safety evaluation

Regulatory guidelines for first-in-human trials subject to regular review

Access to information from unpublished studies

Sharing of safety information from phase I trials

Preparation and review of clinical trial applications

Communication between sponsors and regulators at an early stage of development

Appraisal of risk by regulators should include consultation from outside experts

Ample time for reviewing complex cases

\section{Design of the phase I trial}

Special consideration should be given to new agents for which the primary mode of action cannot be evaluated in animals

Calculation of starting dose should consider a variety of factors, not just the "no-observable-effect level in animals"

Selection of starting doses should err on the side of caution

Consideration of rate of administration

Trial design should be appropriate for the drug

New agents should be given sequentially with an appropriate period of observation between dosing

Decision to use healthy volunteers or patients in first-in-human studies should be carefully considered and fully justified

\section{Clinical environment}

Qualifications of the principal investigator

Management of risk

Appropriate facilities, equipment, and staff

\section{Developing expertise}

Increase the number of qualified principal investigators

Development of specialized centers for first-in-human studies

Data are from ref. 4.

supervision of PAREXEL International, a contract research organization. This phase I trial was designed to test single i.v. doses of TGN1412 in four different dosage cohorts: $0.1,0.5,1.0$, and $2.0 \mathrm{mg} / \mathrm{kg}$. The randomization scheme specified a $3: 1$ ratio of active drug to placebo and a sample size of eight subjects for each dosage cohort. Beginning at 8:00 am on day 1, each of the volunteers received an i.v. infusion of TGN1412 or placebo that lasted 3-6 minutes. The subjects received the infusions in succession, 10 minutes apart. As previously described by Suntharalingam et al. (1), all six of the volunteers receiving TGN1412 developed a systemic inflammatory response within 90 minutes that was characterized by the rapid induction of circulating proinflammatory cytokines and the early appearance of headache, myalgia, nausea, diarrhea, erythema, vasodilation, and hypotension. The six volunteers became critically ill within the next $12-24$ hours, manifesting pulmonary infiltrates and injury, renal failure, and disseminated intravascular coagulation. They were so ill as to require mechanical ventilation, dialysis, and infusion of fresh frozen plasma and cryoprecipitate to correct the coagulopathy. Additional treatment consisted of high-dose methylprednisolone and an anti-IL- 2 receptor antibody. By 8 hours after the infusion, all of the six TGN1412treated patients had developed profound lymphopenia, with nadirs at 24 hours, followed by slow recovery in $\mathrm{T}$ cell numbers over the next 1-2 weeks. In the first hour, dramatic increases were measured in the serum levels of TNF- $\alpha$ followed shortly thereafter by large increases in IFN- $\gamma$ and IL-10. By the end of day 2, the cytokine storm had largely subsided and the volunteers had evolving multi-organ failure that ultimately lasted about 1-2 weeks, with gradual clinical improvement over the next 2-4 weeks. Regulatory authorities subsequently tested the batch of TGN1412 used in this trial and found no contamination with endotoxin or errors in manufacturing or administration, indicating that the observed cytokine storm was caused by the ligation of CD28 on human cells.

\section{Call to action}

What have we learned from this unfortunate mishap? Shortly after this tragedy,
Kenter and Cohen raised a series of issues concerning the analysis of risk for new compounds for clinical development (8). The Medicines and Healthcare Products Regulatory Agency (MHRA) also conducted a thorough evaluation. The MHRA found no discrepancies at PAREXEL after inspecting their facilities, equipment, quality systems, and documentation. An assessment of the manufacturing process confirmed that the product met all of the batch release criteria. An Expert Scientific Group (ESG) was convened to consider what factors should be considered before first-in-human studies of agents are conducted with novel mechanisms of action. The ESG made 22 recommendations, encompassing various aspects of the drug development process (9). In their totality, these recommendations touch on the obligations of the sponsor, the appraisal of high-risk agents such as TGN1412, the qualifications and scientific expertise of the investigator, and strategies to minimize risk in first-in-human trials (see Issues raised in the ESG report related to first-in-human studies).

One aspect of the ESG report (9) dealt with the safety profile for novel agents as 
it relates to the science behind the putative mechanism of action and the need for indepth review of this body of information by independent experts with research knowledge in the field. Regulators would have been justified in asking for more information about the safety of TGN1412 than available in the clinical trial application, including more analysis about the effects of an untested mechanism of action in man. Nine of the 22 recommendations put forward by the ESG related to study design, including initial dosing of drug. Without a doubt, TGN1412 should have been considered a high-risk drug, mandating a much lower starting dose than was selected for the first-in-human trial. While it will probably never be known if a lower starting dose would have attenuated the serious adverse events observed in this trial, such a cautionary approach appears to be warranted given the potential for TGN1412 to stimulate massive $\mathrm{T}$ cell activation.

Another recommendation called for new agents to be given sequentially, with an appropriate observation period between dosing. Ten minutes is simply too short an interval between dosing to observe for infusion-related adverse events, and a longer period of observation for each subject would have saved the other five volunteers in the TGN1412 trial from suffering the same fate as the first subject who received the drug. Finally, the ESG raised the issue of the qualifications of investigators that participate in first-in-human studies. They proposed the development of a national accreditation system for principal investigators involved in first-in-human studies. A trained investigator would not have likely accepted the study design for the TGN1412 trial as written, which affords another check in the system that might have minimized the severity of the adverse events. Although the government regulatory bodies are the final arbitrator in the system, the whole process would be enormously aided by a structured set of principles for evaluating high-risk agents in humans that was available to the sponsor, investigator, and other relevant research personnel as well as ethics boards.

\section{Probing for answers}

Since these tragic events, additional studies have been performed in an attempt to better understand the causes of the cytokine storm and the other immunological outcomes of TGN1412 treatment. Conspicuously lacking in the TGN1412 preclini- cal package was information about the effects of this antibody on human T cells. Warning bells would have likely sounded in the minds of the regulators if they had known that a superagonistic anti-human CD28 antibody induced rapid depletion of peripheral $\mathrm{T}$ cells in mice with a humanized immune system (10). We now know about these studies, as they were published after the fact. How to design experiments in vitro to predict the effects of a targeted immunotherapeutic in vivo remains a fundamental question for drug developers. To pinpoint the shortcomings in the preclinical testing, Stebbings et al. (11) set up six different protocols for presenting TGN1412 to human PBMCs or cynomolgus monkey PBMCs in vitro. They found that when TGN1412 (1.0 and 10.0 $\mu \mathrm{g} /$ well) was air dried on wells and added to human PBMCs, it induced the release of large amounts of TNF- $\alpha$, IL- 6 , and IL-8. Also, a similar cytokine release profile was obtained when TGN1412 (0.1, 1.0, and $10 \mu \mathrm{g} /$ well) was added in aqueous solution to a coculture of human PBMCs and endothelial cells.

Interestingly, they could not duplicate these responses using PBMCs from cynomolgus monkeys, suggesting that TGN1412 does not behave the same in nonhuman primate species as in humans. One reason this result may not be so surprising is because the extracellular domain of human CD28 differs by four amino acids from the macaque CD28 sequence, including a G68E substitution in the C'D binding loop (i.e., the site of superagonistic anti-CD28 monoclonal antibody binding). Since TGN1412 presented to endothelial cells elicited such a powerful cytokine response, it has also been suggested that costimulatory ligands expressed on the human but not the monkey vasculature may have synergized with CD28 costimulation to induce the massive release of proinflammatory cytokines (4). Another possible explanation may derive from the recent observation that human $\mathrm{T}$ cells express little to none of the CD33-related siglecs, which are inhibitory signaling molecules on the cell surface that downregulate cellular activation pathways (12). In contrast, chimpanzees and monkeys express abundant levels of CD33-related siglecs on the surface of their $\mathrm{T}$ cells, which also show lower amounts of $\mathrm{T}$ cell proliferation and activation than their human counterparts upon stimulation with anti-CD3 and antiCD28 antibodies.

\section{CD28 superagonist-induced} T lymphopenia: new insights

In this issue of the JCI, Müller et al. (5) have turned again to rats in an effort to uncover why the $\mathrm{T}$ cell activation, profound lymphopenia, and dramatic cytokine storm induced by TGN1412 treatment in humans was not anticipated on the basis of earlier animal experiments in the preclinical phase of TGN1412's development. These authors found that i.v. infusion of rats with JJ316, the rat functional equivalent of TGN1412 that had been instrumental in most of the relevant preclinical studies, caused a rapid redistribution of all $\mathrm{CD}^{+} \mathrm{T}$ cells from the periphery to the spleen and lymph nodes (5), and therefore, simulated the $\mathrm{T}$ cell lymphopenia observed in humans following TGN1412 administration. The authors showed, via intravital video microscopy, that $\mathrm{T}$ cell motility was dramatically reduced immediately following TGN1412 administration, suggesting that the observed $T$ cell redistribution was a consequence of $T$ cells becoming trapped in the secondary lymphoid organs. Additional factors contributing to impaired $\mathrm{T}$ cell recirculation may also include the observed increase in $T$ cell size, changes in the $T$ cell cytoskeleton, and increased $\mathrm{T}$ cell adhesion to fibronectin. Müller et al. went on to show that, in addition, JJ316 impaired T cell egress from the spleen and lymph nodes via the downregulation of the sphingosine 1phosphate receptor EDG-1 that is predominantly expressed on T cells. As such, cell-cell and cell-matrix interactions in combination with impaired $\mathrm{T}$ cell egress appear to play a role in the $\mathrm{T}$ lymphopenia, resulting from superagonistic antiCD28 antibody treatment.

In addition to these proadhesive effects, Müller et al. found that JJ316 infusion induced two distinct waves of $\mathrm{T}$ cell activation (5). The first wave of $\mathrm{T}$ cell activation and redistribution was characterized by elevated levels of proinflammatory cytokines, including IL-17 and IFN- $\gamma$ as well as increased expression of the cell surface markers CD25, CD69, and CD134. Surprisingly, JJ316 infusion elicited only mildly elevated levels of TNF- $\alpha$ and IFN- $\gamma$ in the serum of treated rats, which produced no discernable clinical effects and were several orders of magnitude below that seen in the TGN1412-treated subjects. Therefore, JJ316 administration in rats only partially mimics the effects of TGN1412 in humans. Consequently, Mül- 
ler et al. suggest that it is the secretion rather than the synthesis of these proinflammatory mediators that may account for the differences in the reaction of rats versus humans to superagonistic antiCD28 antibodies and may account for why the cytokine storm observed in humans receiving TGN1412 could not have been predicted based on preclinical work in rodents receiving JJ316.

The first wave of $\mathrm{T}$ cell activation disappeared within 48 hours and was followed by a second wave of expansion of $\mathrm{CD}^{+} \mathrm{CD}_{25}{ }^{+} \mathrm{FoxP}^{+}$Tregs (5), involving Treg enlargement, polarization, and increased motility. The authors suggest that the cytokine storm observed in TGN1412-treated individuals was likely a consequence of the first wave of $\mathrm{T}$ cell activation and that the beneficial effects of superagonistic anti-CD28 antibody therapy previously observed in rodent models of autoimmune disease were likely the result of this second wave of activation that selectively affects Tregs. In summary, Müller et al. further illuminate our understanding of the mechanisms of action of the superagonist anti-CD28 antibody, and the data reinforce that this therapeutic approach will require much further investigation before it can be applied to humans.

\section{Inspiring trust in drug development}

Human volunteers who chose to participate in clinical trials trust in our ability to protect them as much as possible from the dangers of investigational agents. Trust is not an intangible quality but rather something real and concrete. Trust is not only about integrity but also about competence. People expect investigators, sponsors, contract research organizations, and regulatory authorities to have the talents, skills, knowledge, and capacity to carry out their responsibilities. We may lose their trust if we fail to meet our commitments to afford optimal protection of human subjects in trials. Participation in clinical trials will always have inherent risks, but hopefully lessons will be learned from the TGN1412 experience that will benefit research subjects in the future.

Address correspondence to: E. William St. Clair, Department of Medicine, Box 3874, Duke University Medical Center, Durham, North Carolina 27710, USA. Phone: (919) 684-4499; Fax: (919) 684-8358; E-mail: stcla003@mc.duke.edu.

1. Suntharalingam, G., et al. 2006. Cytokine storm in a phase I trial of the anti-CD28 monoclonal antibody TGN1412. N. Engl. J. Med. 355:1018-1028.

2. Beyersdorf, N., et al. 2005. Selective targeting of regulatory $\mathrm{T}$ cells with $\mathrm{CD} 28$ superagonists allows effective therapy of experimental allergic encephalomyelitis. J. Exp. Med. 202:445-455.

3. Schmidt, J., et al. 2003. Treatment and prevention of experimental autoimmune neuritis with superagonistic CD28-specific monoclonal antibodies. J. Neuroimmunol. 140:143-152.

4. Rodríguez-Palmero, M., et al. 2006. Effective treatment of adjuvant arthritis with a stimulatory CD28-specific monoclonal antibody. J. Rheumatol. 33:110-118.

5. Müller, N., et al. 2008. A CD28 superagonistic antibody elicits 2 functionally distinct waves of T cell activation in rats. J. Clin. Invest. 118:1405-1416.

6. Hünig, T. 2007. Manipulation of regulatory T-cell number and function with CD28-specific monoclonal antibodies. Adv. Immunol. 95:111-148.

7. TeGenero AG Investigator's Brochure. TGN1412 humanized agonistic anti-CD28 monoclonal antibody. Edition 1.1. http://www.circare.org/foia5/ $\operatorname{tgn} 1412$ investigatorbrochure.pdf.

8. Kenter, M.J.H., and Cohen, A.F. 2006. Establishing risk of human experimentation with drugs: lessons from TGN1412. Lancet. 368:1387-1391.

9. Dowsing, T., and Kendall, M.J. 2007. The Northwick Park tragedy - protecting healthy volunteers in future first-in-man trials. J. Clin. Pharm. Ther. 32:203-207.

10. Legrand, N., et al. 2006. Transient accumulation of human mature thymocytes and regulatory $\mathrm{T}$ cells with CD28 superagonist in "human immune system" Rag2 $2^{-/-\gamma_{c}-/-}$ mice. Blood. 108:238-245.

11. Stebbings, R., et al. 2007. "Cytokine storm" in the phase I trial of monoclonal antibody TGN1412: better understanding the causes to improve the preclinical testing of immunotherapeutics. J. Immunol. 179:3325-3331.

12. Nguyen, D.H., et al. 2006. Loss of siglec expression on T lymphocytes during human evolution. Proc. Natl. Acad. Sci. U. S. A. 103:7765-7770.

\title{
Tetraspanin in oncogenic epithelial-mesenchymal transition
}

\author{
Ruth J. Muschel and Annamaria Gal
}

Radiation Oncology and Biology, The Radiobiology Research Institute, University of Oxford, Oxford, United Kingdom.

\begin{abstract}
Members of the L6 family of membrane proteins, a branch of the tetraspanin superfamily, are overexpressed in tumor cells from many types of cancers. However, direct evidence of their oncogenic activity has not been previously shown. In this issue of the JCI, Lee et al. demonstrate that overexpression of the tetraspanin superfamily member TM4SF5 in human hepatocellular carcinoma cells causes cellular phenotypic changes that resemble classical descriptions of epithelial-mesenchymal transition (EMT), with some unique aspects (see the related article beginning on page 1354). They also show that these TM4SF5-mediated effects trigger tumor formation when these cells are injected into mice. The study implicates TM4SF5, for the first time to our knowledge, in EMT oncogenic pathways of cancer progression.
\end{abstract}

Nonstandard abbreviations used: EMT, epithelialmesenchymal transition; TM4SF5, transmembrane 4 L6 family member 5; ZO-1, zonula occludens-1.

Conflict of interest: The authors have declared that no conflict of interest exists.

Citation for this article: J. Clin. Invest. 118:1347-1350 (2008). doi:10.1172/JCI35308.
Over many years, cancer researchers have attempted to unlock the secrets of cancer cells by comparing the gene expression of tumor cells to that of their normal cellular counterparts. The genes so identified have in many cases proven to be important mediators of the transformed phenotype and have led in a few cases to the development of clinically useful therapeutics, such as antibodies directed against EGFR (implicated in many types of epithelial cancers) or human EGFR 2 (HER2/neu; often overexpressed in breast cancer). In 1997, Gress et al. performed a large-scale screen for differentially expressed genes in tissue from individuals with pancreatic cancer compared with tissue from individuals with chronic pancreatitis and identified transmembrane 4 L6 family member 5 (TM4SF5) as a gene upregulated in pancreatic tumors (1). TM4SF5 was noted to be homologous to the integral membrane protein $\mathrm{L} 6$ that is also overexpressed in a variety of malignant tissues (2). 